\title{
Thermal Effect on E/M Impedance Spectroscopy of Piezoelectric Wafer Active Sensors
}

\author{
Tuncay Kamas*a, Erik Frankforter ${ }^{\mathrm{b}}$, Bin Lin ${ }^{\mathrm{b}}$, Lingyu (Lucy) Yu ${ }^{\mathrm{b}}$, Victor Giurgiutiu ${ }^{\mathrm{b}}$ \\ ${ }^{a}$ Mechanical Engineering Department Osmangazi University, Meselik Campus, Eskişehir, \\ Türkiye 26480 \\ ${ }^{\mathrm{b}}$ Mechanical Engineering Department University of South Carolina, 300 Main Street, \\ Columbia, SC, USA 29208
}

\begin{abstract}
This paper presents theoretical predictive modeling and experimental evaluation of the structural health monitoring capability of piezoelectric wafer active sensors (PWAS) at elevated temperatures. Electromechanical impedance spectroscopy (EMIS) method is first qualified using circular PWAS resonators under traction-free boundary condition and in an ambience with increasing temperature. The theoretical study is conducted regarding temperature dependence of the electrical parameters, the capacitance $\mathrm{C}_{0}, \mathrm{~d}_{31}$ and $\mathrm{g}_{31}$; and the elastic parameters, the in-plane compliance $s_{11}$ and Young's modulus $c_{11}$, of piezoelectric materials. The Curie transition temperature must be well above the operating temperature; otherwise, the piezoelectric material may depolarize under combined temperature and pressure conditions. The material degradation is investigated by introducing the temperature effects on the material parameters that are obtained from experimental observations as well as from related work in literature. The preliminary results from the analytical 2-D circular PWAS-EMIS simulations are presented and validated by the experimental PWAS-EMIS measurements at elevated temperatures. Temperature variation may produce pyro-electric charges, which may interfere with the piezoelectric effect. Therefore, analytical simulations are carried out to simulate the pyro-electric response from the temperature effects on a free circular PWAS-EMIS in in-plane mode. For the experimental validation, PWAS transducers are placed in a fixture that provides the traction-free boundary condition. The fixture is then located in an oven integrated with PID temperature controller. The EMIS measurement is conducted during the temperature increase and the first resonance frequency peak in admittance and impedance spectra was acquired.
\end{abstract}

Keywords: PZT resonator, E/M impedance, thermal effects, PZT material degradation, analytical prediction

\section{INTRODUCTION}

The thermal effects at elevated temperatures mostly exist for structural health monitoring (SHM) applications. The technologies for diagnosis and prognosis of SHM systems need to take the thermal effect into account and compensate it on sensing and in-situ monitoring of structures. One of the extensively employed sensor technologies has been permanently installed piezoelectric wafer active sensor (PWAS) for in-situ continuous SHM.

Piezoelectric wafer active sensor (PWAS) $)^{1,2}$ is light-weighted, inexpensive, unobtrusive, minimally intrusive sensor requiring low-power. PWAS is made of piezoelectric ceramic with electric field polarization, $E_{3}$, across the electrodes deposited on both surfaces. Using the transduction of ultrasonic elastic waves into voltage and vice versa, PWAS has been emerged as one of the major SHM sensing technologies and non-destructive evaluations (NDE) ${ }^{3,4}$. However, the dynamic characteristics of PWAS need to be explored prior its installation for in-situ SHM. Electro-mechanical impedance spectroscopy (EMIS) method has been utilized as a dynamic descriptor of PWAS and as a high frequency local modal sensing technique by applying standing waves to indicate the response of the PWAS resonator by determining the resonance and anti-resonance frequencies for in-situ ultrasonics ${ }^{5}$ such as in the work presented by Sun and Liang ${ }^{6,7}$. They utilized the EMIS method for high frequency local modal sensing.

Sensors and Smart Structures Technologies for Civil, Mechanical, and Aerospace Systems 2015,

edited by Jerome P. Lynch, Kon-Well Wang, Hoon Sohn, Proc. of SPIE Vol. 9435, 943500

(C) 2015 SPIE · CCC code: $0277-786 X / 15 / \$ 18 \cdot$ doi: $10.1117 / 12.2083958$

Proc. of SPIE Vol. 9435 943500-1 
A literature survey is first conducted to assess prior work on the survivability of piezoelectric wafer active sensors (PWAS) at elevated temperature so that we could discover from the literature the extent of temperature dependence of the electric parameters, i.e. $d_{31}$ and $g_{31}$, and the elastic parameters, i.e. $s_{11}$ and Young's modulus $\left(\mathrm{c}_{11}\right)$, of different piezoelectric materials.

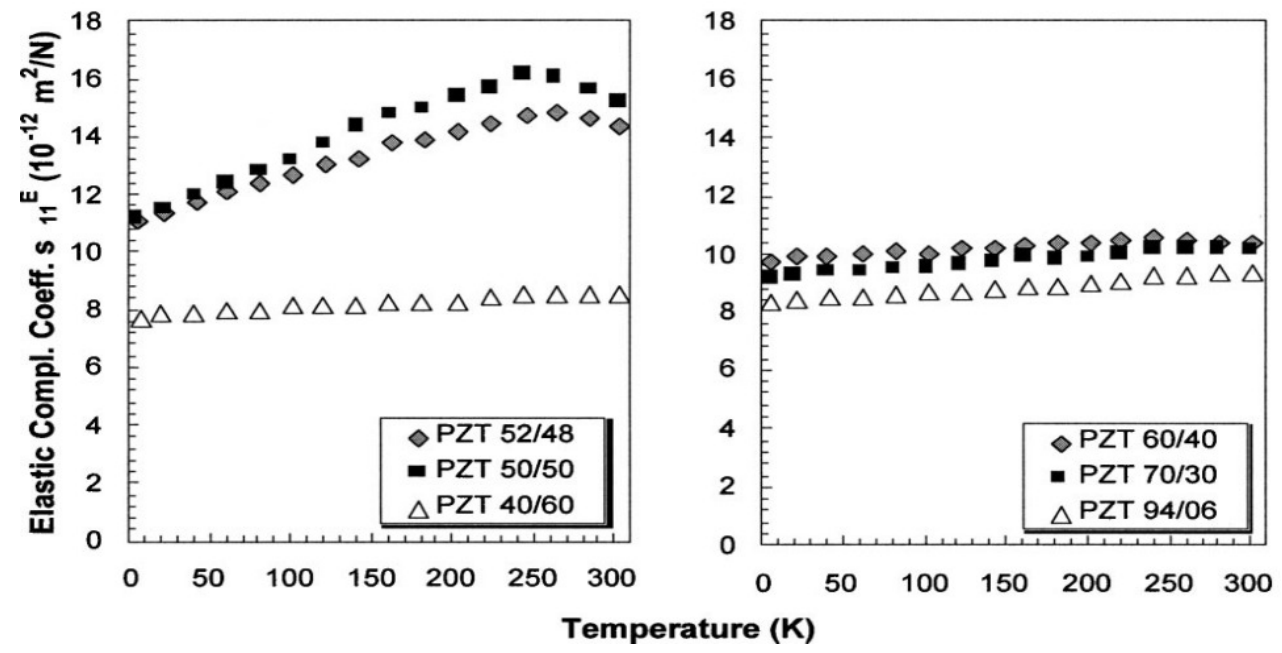

Figure 1 Low temperature elastic compliance coefficient $\left(\mathrm{s}_{11}{ }^{\mathrm{E}}\right)$ plotted as a function of temperature for several tetragonal and rhombohedral PZT compositions.

Wolf (2004) reported increasing piezoelectric elastic compliance upto $250 \mathrm{~K}$ for PZT 52/48 and PZT 50/50 and their compliance values start decreasing after $250 \mathrm{~K}$ however other PZT material with different $\mathrm{Zr} / \mathrm{Ti}$ compositions have monotonic increase until $300 \mathrm{~K}$ as seen in Figure 1.

Raghavan \& Cesnik in 2008 reported elastic and electric properties of a piezoelectric material, PZT-5A as a function of temperature raised up to $150{ }^{\circ} \mathrm{C}$ as seen in Figure 2. The inverse of Young's modulus, Y, is the elastic compliance, i.e.

$$
s=\frac{1}{Y}=\frac{\text { Strain }}{\text { Stress }}\left[\mathrm{m}^{2} / \mathrm{N}, 1 / \mathrm{Pa}\right]
$$
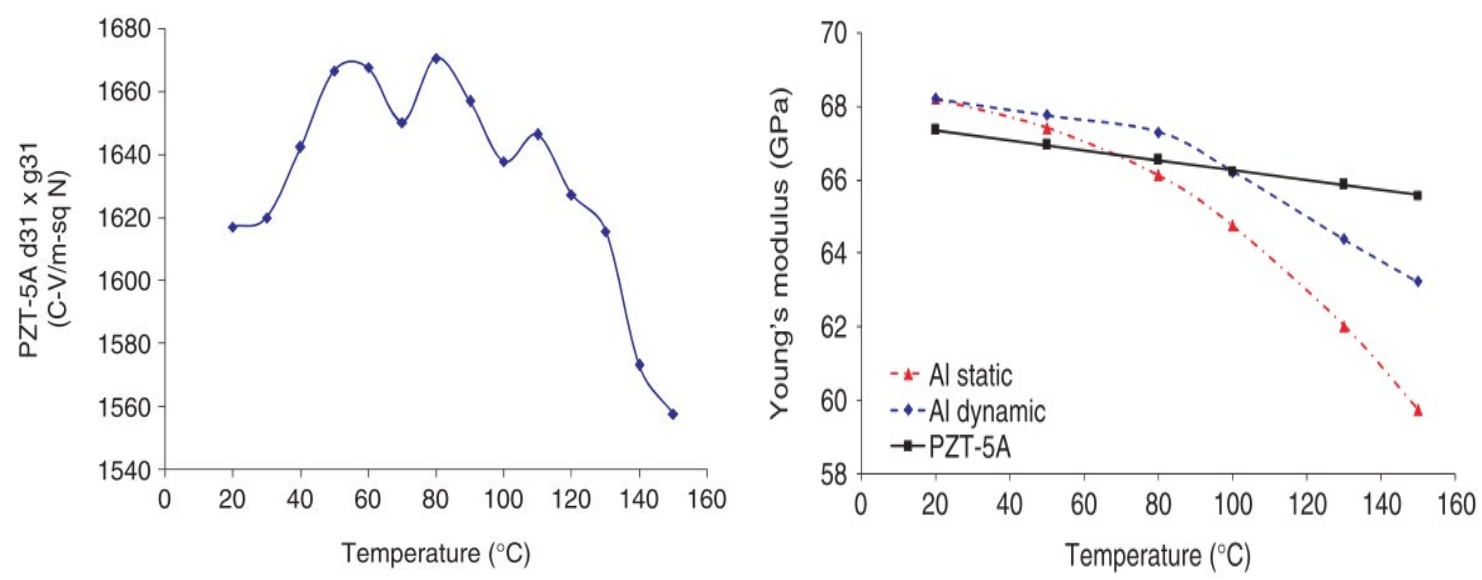

Figure 2 Variation of Young's modulus and $d_{31} x_{31}$. Average thermal expansion for PZT-5A $\alpha_{\text {PZT-5A }}=2.5 \mu m / \mathrm{m}^{\circ}{ }^{\circ} \mathrm{C}$. 
Young's moduli of PZT-5A monotonically decreases as temperature increases between room temperature and $160^{\circ} \mathrm{C}$. The product of $\mathrm{d}_{31} \mathrm{xg}_{31}$ fluctuates along the temperature. It first monotonically inclines until $60^{\circ} \mathrm{C}$, and it declines after $110^{\circ} \mathrm{C}$ and it goes lower than its original value at room temperature.

This paper presents a theoretical and experimental study of the structural health monitoring capability of PWAS at elevated temperatures. Electro-mechanical impedance spectroscopy (EMIS) method is qualified using PWAS at increasing temperature. The material degradation is investigated by introducing the temperature effects on some parameters. The results from the analytical PWAS-EMIS simulations are presented. Temperature variation may produce pyroelectric charges, which may interfere with the piezoelectric effect. Therefore, the analytical model is extended by implying the pyroelectric charge term into the piezoelectric constitutive equations to simulate the temperature effects on a free circular PWAS-EMIS. For the experimental validation, the EMIS measurements are conducted during the temperature increase. Then, the results from the experimental cycling of PWAS at gradually increasing temperatures are discussed. Trends of the results in terms of static capacitance, $\mathrm{C}_{0}$ and electromechanical impedance spectroscopy (EMIS) are presented.

\section{ANALYTICAL EMIS SIMULATIONS FOR PWAS AT ELEVATED TEMPERATURES}

In this subsection, the temperature effects on free circular PWAS admittance and impedance are presented through the analytical model and the EMIS tests. The effects of the stiffness coefficient $\mathrm{c}_{11}$, the piezoelectric coefficient $\mathrm{d}_{31}$, and the static capacitance $\mathrm{C}_{0}$ on impedance/admittance are taken into account.

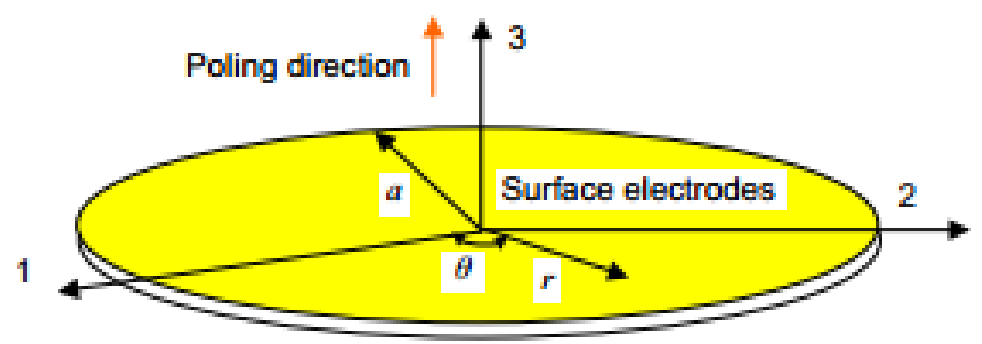

Figure 3 Schema of circular PWAS in cylindrical coordinate system.

Zagrai ${ }^{10}$ has developed 2-D EMIS for circular PWAS using the free circular PWAS model (Figure 4.11) and the derivation procedure shown in the flow-chart in Figure 4. In this section, we adopted herein his in-plane EMIS model to simulate the temperature effects on piezoelectric material degradation of free circular PWAS. The analytical simulation will be conducted by changing the stiffness coefficient, the piezoelectric coefficient, and the capacitance. The stiffness coefficient and the piezoelectric coefficient degradation have been discussed in the literature and plots for the material properties versus temperature increase have been provided. The capacitance dependence over temperature has been defined during our experimental studies. Therefore, the proportions of the elastic and piezoelectric material property degradations are attained from the literature and the capacitance proportion was obtained from the capacitance measurements over increasing temperature. The admittance and impedance simulations are presented respectively and compared with the experimental results. 


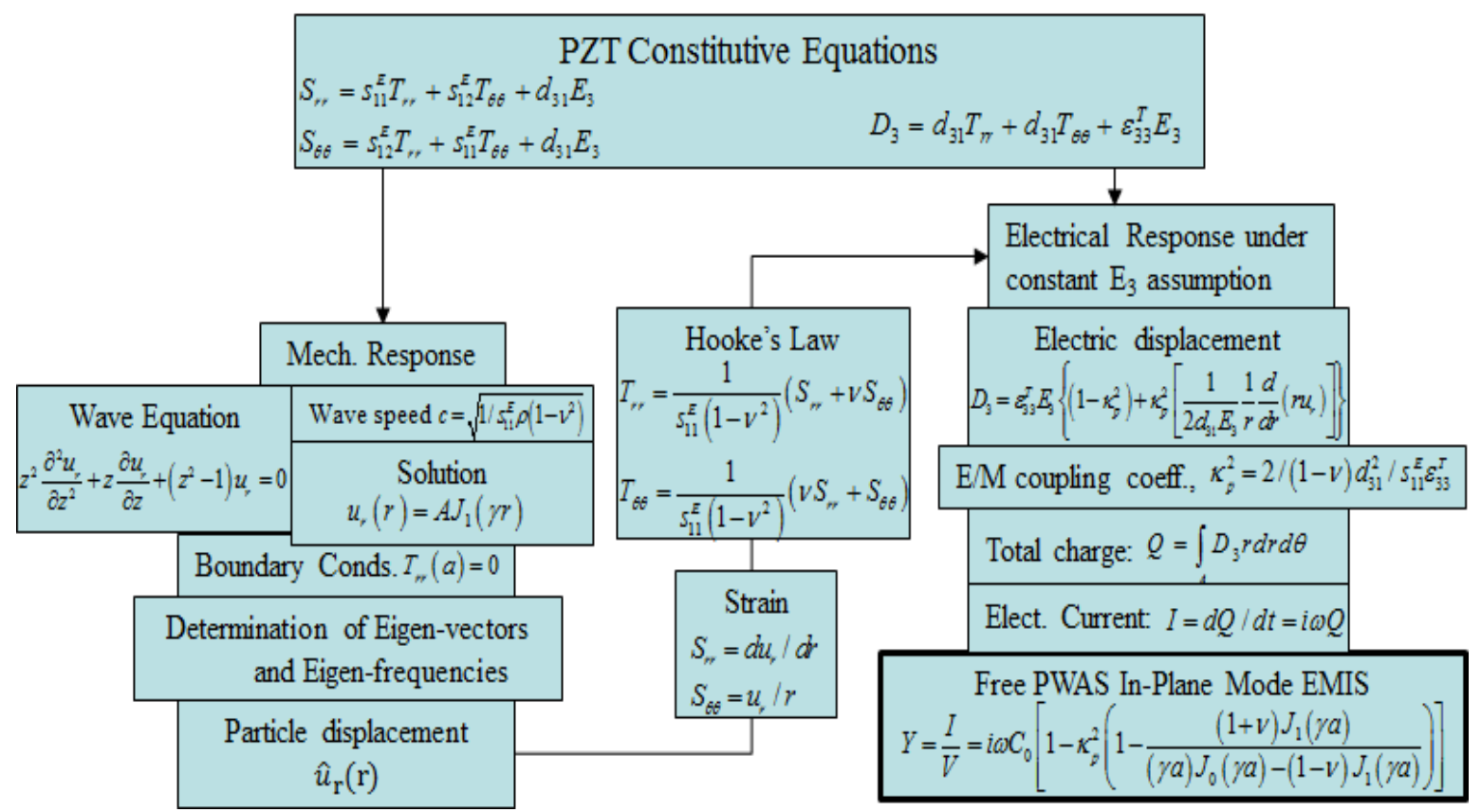

Figure 4 Flow-chart of the analytical modeling of 2-D in-plane EMIS of circular PWAS.

\section{EXPERIMENTAL WORK}
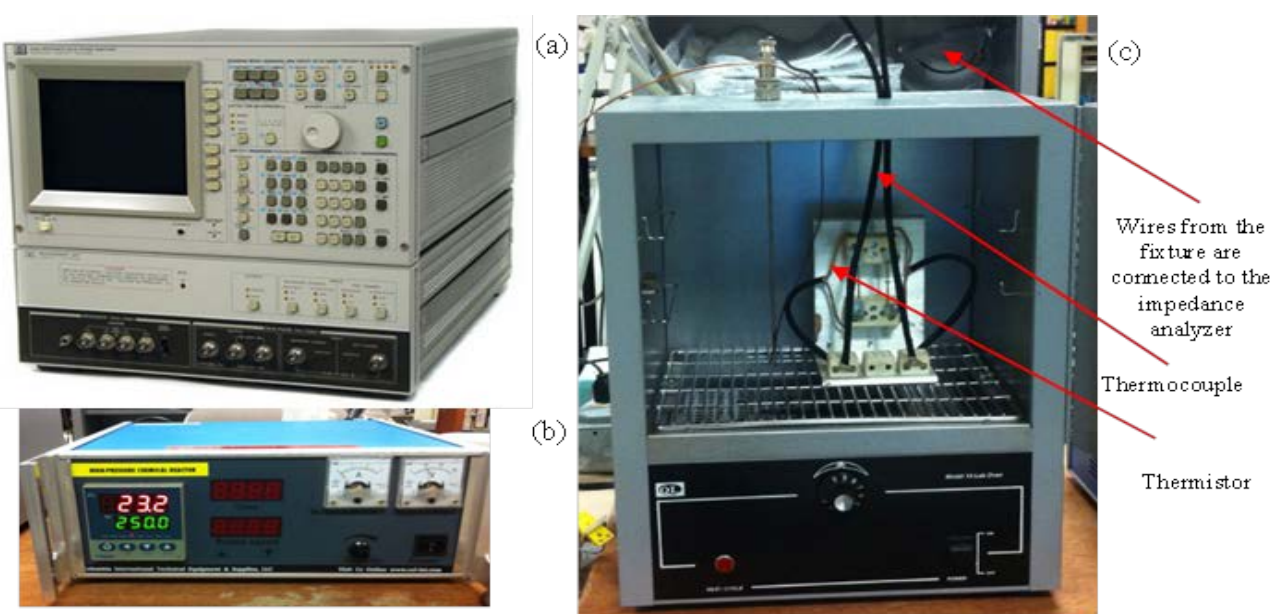

Figure 5 High temperature PWAS testing by (a) impedance analyzer, (b) PID temperature controller (c) oven.

The E/M impedance is used as a direct and convenient method to implement for PWAS impedance signature as a function of temperature up to relatively high temperature, the required equipment being an electrical impedance analyzer, such as HP 4194A impedance analyzer, PID temperature controller, and oven. An example of performing PWAS E/M impedance spectroscopy is presented for PWAS located in a fixture in the oven in Figure 5. PWAS has to have stress-free i.e. unconstrained boundary conditions so that it was fixed by pogo-pins that only apply low spring forces point-wise on the PWAS surfaces in the fixture. The fixture has wires that can be connected with the probes of the EMIS analyzer instrument. The impedance analyzer reads the E/M impedance of PWAS itself in the oven. It is applied by scanning a predetermined frequency range $(300 \mathrm{kHz}-$ $400 \mathrm{kHz}$ ) and recording the complex impedance spectrum. A LabView data acquisition program was used to control the impedance analyzer and sweep the frequency range in steps (of $100 \mathrm{~Hz}$ ) that was predefined and to attain the data in a format that assists to data analysis. During the visualization of the frequency sweep, the real part of the E/M impedance, $\operatorname{Re}(Z(\omega))$, follows up and down variation as the structural impedance goes through the peaks and valleys of the structural resonances and anti-resonances. 
Our objective was to evaluate the extent of the temperature dependence of PWAS-capacitance and EMIS results and the degredation of PWAS material with respect to some electrical and elastical material properties. For the experimental study, we pursued the following protocol

- Measure baseline room temperature PWAS capacitance and electromagnetic impedance (EMI) spectrum.

- $\quad$ Elevate PWAS to $50{ }^{\circ} \mathrm{C}$ and hold it there for several minutes (e.g. 10-30 min)

- $\quad$ Measure PWAS capacitance and EMIS at the elevated temperature

- $\quad$ Drop PWAS temperature back to the room temperature

- $\quad$ Measure PWAS capacitance and EMI spectrum at room temperature

- Perform steps $2-5$ for $100,150,200$, and $250^{\circ} \mathrm{C}$

We have measured 6 PWAS at elevated temperatures starting from the room temperature. The capacitance and EMIS measurements have been performed for $50,100,150,200$, and $240^{\circ} \mathrm{C}$ and the temperature was dropped to the room temperature after each step and obtained 5 temperature cycles and 5 more capacitance and EMIS reading at the room temperatures.

\section{RESULTS AND DISCUSSION}

\subsection{Capacitance results}

Figure 6 illustrates the averaged capacitance values over the static capacitance values that are obtained from 6 PWAS resonators. One can observe the monotonic increase in the capacitance values by increasing the temperature. The trend is linear up to $200{ }^{\circ} \mathrm{C}$ however it is interesting to see that the temperature gradient of the capacitance increases after this temperature. The capacitance values at room temperatures after each cycling also vary for the PWAS 1R.

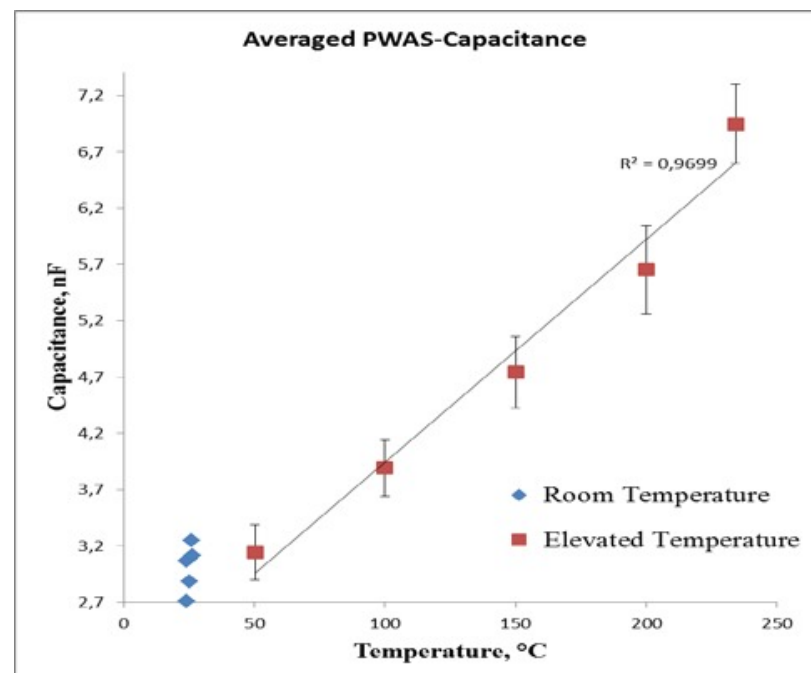

Figure 6 Averaged static capacitance results for all of the circular PWAS resonators $(7 \mathrm{mmx} 0.2 \mathrm{~mm})$ at room temperature and elevated temperatures. 


\subsection{EMIS results}

We have measured 6 PWAS resonators in the high temperature EMIS test. We denoted them by their sequence number and a letter R or $L$ that denotes right or left. Then PWAS $1 \mathrm{R}$ is the first PWAS located on right side of the fixture and 2L is the second PWAS on the left and so on. Moreover, the first room temperature in the cycle is denoted RT1 as the second room temperature after cooling down from $50^{\circ} \mathrm{C}$ is denoted RT2 so that the impedance signature for the first PWAS on the right at the first room temperature is represented by 1R_RT1 and the second PWAS on the left at the third room temperature after cooling from $100^{\circ} \mathrm{C}$ is represented by 2L_RT3 and so on.

We will discuss the analyses of impedance results of each PWAS in sequential subsections. We will first plot all the impedance signatures at the first in-plane anti-resonance frequency for one PWAS at all elevated temperature-room temperature cycles with a time series plot that shows each time and date when the particular measurement was performed so one can observe how long the overall measurement has taken and how long a PWAS has been kept at a certain temperature. Next, we will separate the EMIS test results as the impedance plots at room temperatures and at elevated temperatures to see the trends of the frequency and amplitude shifts of the first impedance curves in both cases. Then, we will plot the impedance amplitude vs temperature including those at room temperatures in the same graph and also plot the impedance frequency vs temperature in the same way. Finally, we will present the amplitude and the frequency shifts separately at elevated temperatures and those at room temperatures were plotted by the order number of the room temperature. The overall tests have lasted 2 days because cooling down from high temperatures to the room temperature at each cycle has taken somewhat long time. No forced convection method was implied to quicken the cooling process.

The impedance peaks diminish in amplitude as the temperature increases and it keeps diminishing even PWAS is cooled down to the room temperature in each temperature cycle. Another phenomena is the frequency downshift as the temperature moves up however frequency upshift is also observed as PWAS cools down to the room temperature, the PWAS does not seem to recover completely and its impedance cannot move up to its original anti-resonance frequency. One interesting phenomena is also the deformation on the impedance signature of PWAS-1R at RT6 after cooling it down from $250^{\circ} \mathrm{C}$ as the other impedance signatures are smooth curves before.

Overall the trend seems by investigating the plot however it can be more clearly observed those at room temperatures and the elevated temperatures in separate plots as depicted in Figure 8. We utilized color codes for these two plots. For instance, the impedance curve at the second room temperature (1R_RT2) has the same color as the one at the temperature of $50^{\circ} \mathrm{C}$ since it is the room temperature after cooling down from that temperature. The color codes are implied in such order to the other impedance curves.
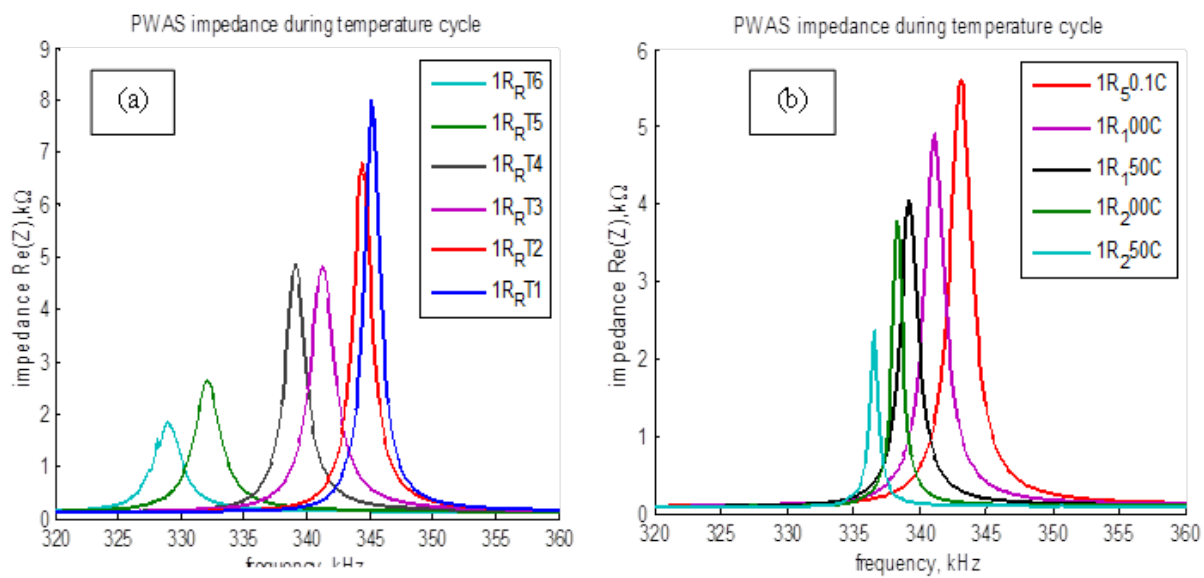

Figure 7 Impedance signature of PWAS-1R (7mmx0.2mm PWAS) at (a) elevated temperatures and (b) room temperatures in each temperature cycle.

In the first cycle, from the room temperature to $50^{\circ} \mathrm{C}$ and back to the room temperature, the impedance frequency first declines then inclines back to the close value although this does not occur in the next two cycles between the room temperature and the $100^{\circ} \mathrm{C}$ and the $150^{\circ} \mathrm{C}$ tests. In the test results from the temperature cycle between the room temperature and $100^{\circ} \mathrm{C}$, the impedance frequency remains the same as the PWAS cooled down 
to the room temperature, which also occurs in the cycle between the room temperature and the $150^{\circ} \mathrm{C}$. More significantly, in the results from the next two temperature cycles, the impedance frequency declines even further as the PWAS cools down back to the room temperature. The PWAS behaves in different manner after it has been heated up to $200^{\circ} \mathrm{C}$ due to the material degradation as the temperature approaches to the Curie temperature of the piezoelectric material and due to the depolarization that may occur.
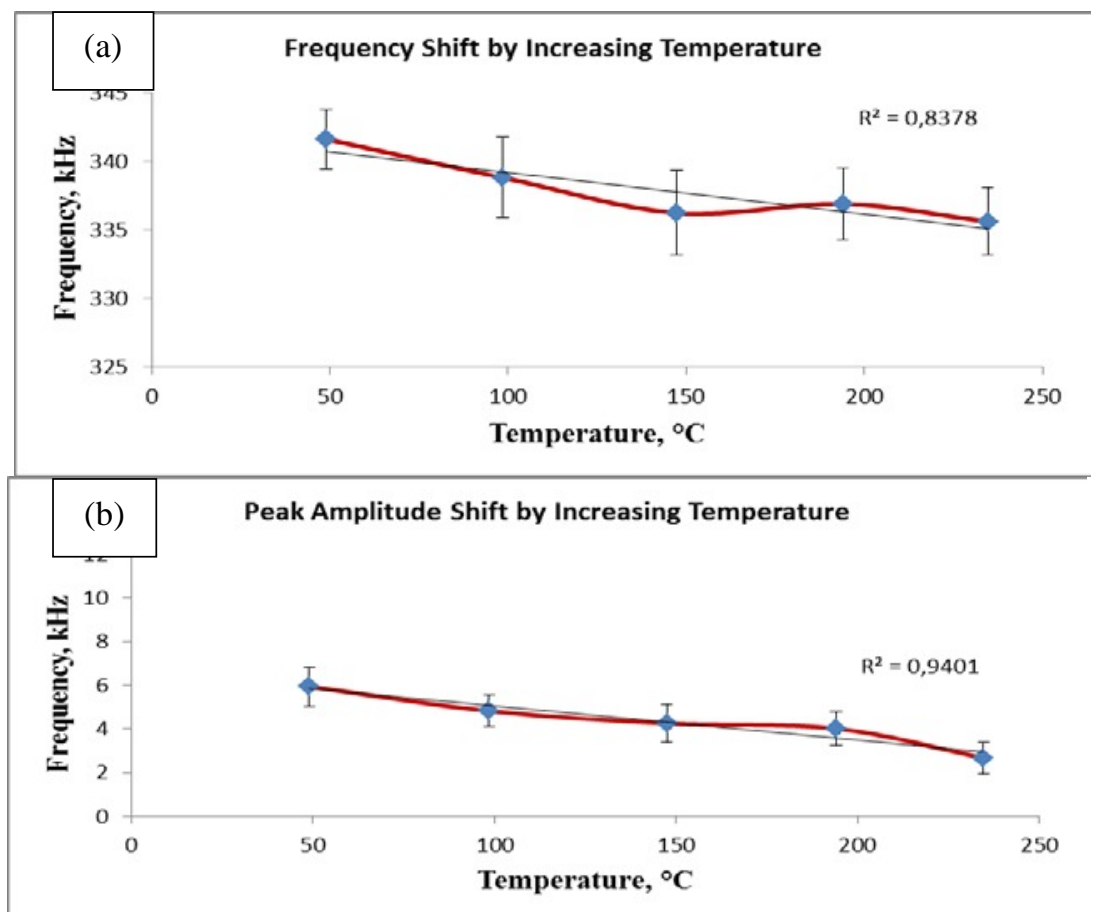

Figure 8 Averaged impedance (a) frequency and (b) ampllitde shift against elevated temperature.

The impedance peak amplitude and frequency against increasing temperature are plotted not including the room temperatures in Figure 9 to analyze the trend of the amplitude and the frequency shifts over elevated temperatures.

\subsection{Comparison between analytical simulations and the experiments}

The EMIS tests are conducted for a PWAS in an oven at elevated temperature between $50^{\circ} \mathrm{C}$ and $250^{\circ} \mathrm{C}$ with the $50^{\circ} \mathrm{C}$ step. During these tests, the piezoelectric material degradation has been observed. The affected material properties are defined via both the literature survey and the measurements. The degraded mechanical, electrical, and piezoelectric properties of PWAS were used to simulate the temperature effects on the first in-plane admittance and impedance peaks. For the analytical simulations, 2-D circular PWAS-EMIS model was utilized. The material properties used in this study are the stiffness coefficient $c_{11}$, the piezoelectric coefficient $d_{31}$, and the capacitance $\mathrm{C}_{0}$. The analytical and experimental results for admittance are shown in Figure 10 and the results for impedance are also shown in Figure 11. 

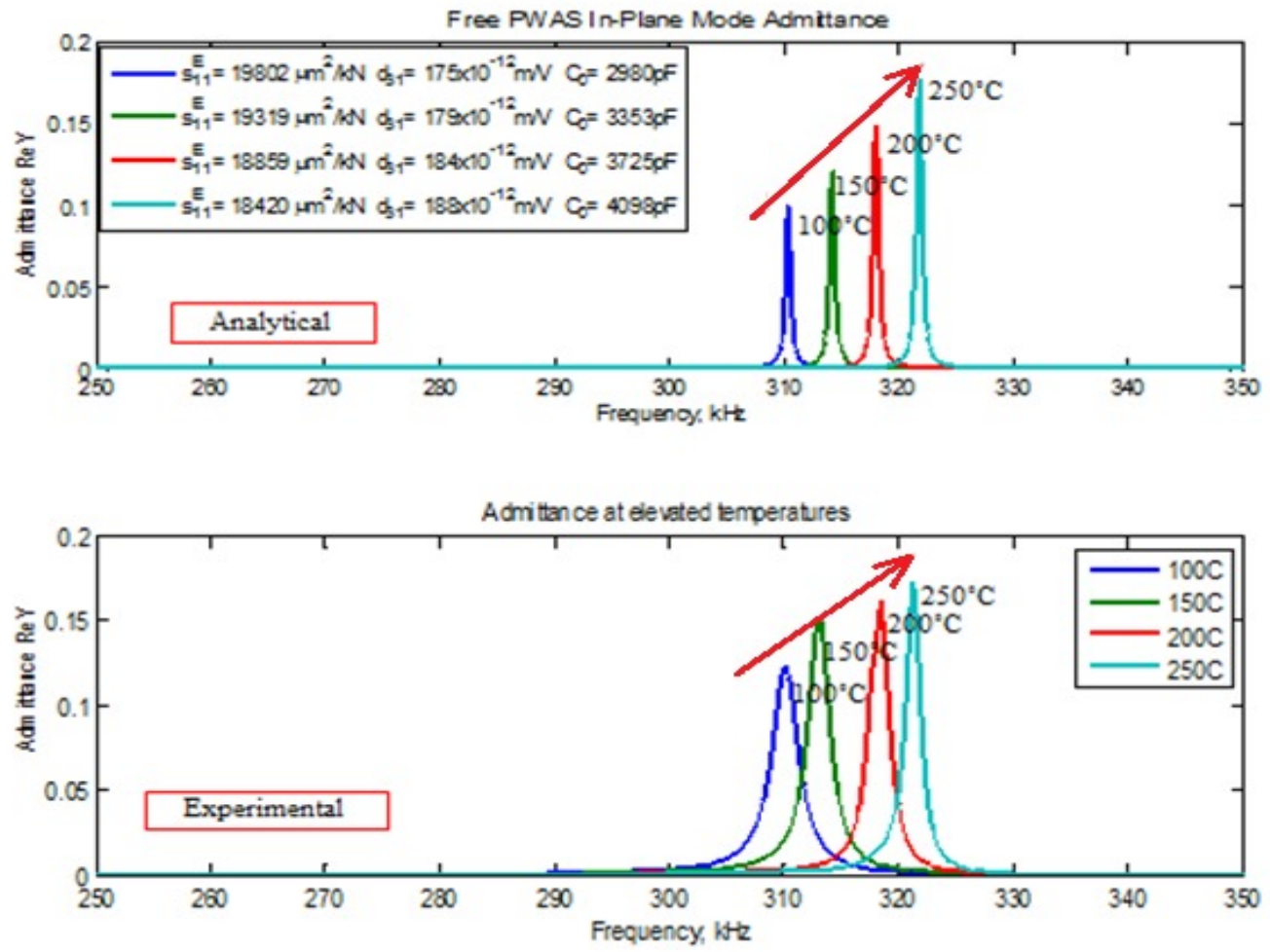

Figure 9 Stiffness coefficient $c_{11}$, piezoelectric coefficient $d_{31}$, and capacitance $C_{0}$ influence on admittance.
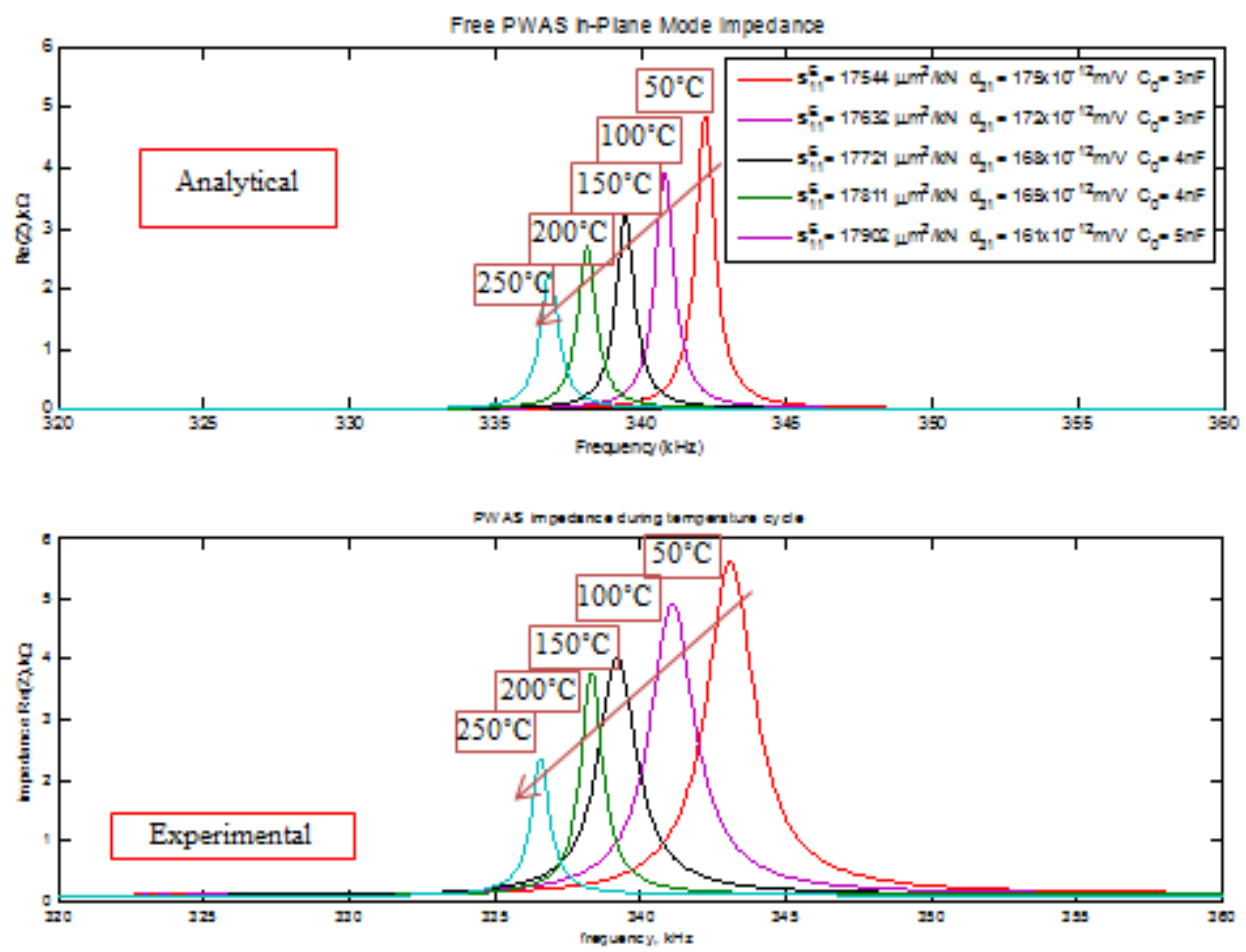

Figure 10 Stiffness coefficient $c_{11}$, piezoelectric coefficient $d_{31}$, and capacitance $C_{0}$ influence on impedance. 


\section{SUMMARY AND CONCLUSIONS}

Past researches were discussed to understand the survivability of piezoelectric wafer active sensors (PWAS) at extreme environments such as at very high temperature etc. Also, we could find out the extent of temperature dependence of the electric parameters, i.e. $\mathrm{d}_{31}$ and $\mathrm{g}_{31}$, and the elastic parameters, i.e. $\mathrm{s}_{11}$ and Young's modulus $\left(\mathrm{c}_{11}\right)$, of different piezoelectric materials.

We have conducted a preliminary parametric study to understand the effects of the material properties on the impedance (anti-resonance) and admittance (resonance) spectra. We utilized the 1-D and 2-D PWAS-EMIS models for the analytical simulations. We have varied the piezoelectric stiffness and the piezoelectric charge constant by $5 \%$. When we combined both the parameter changes and simulate the impedance and admittance of PWAS, we observe both the frequency and the amplitude shifts in both plots. We have phenomenological agreement in trends of the impedance spectra with the experimental results. However, we still need to improve the agreement by using 2-D circular PWAS-EMIS analytical model and by including more parameter changes to reflect the temperature effects on the piezoelectric material degradation. The parameter changes need to be more linked to the temperature increase by using the experimental measurements in the literature. We also need to include in the model the capacitance change that is measured and found to be strongly dependent on the temperature increase.

From the experimental point of view, we observed a linear trend up to $200^{\circ} \mathrm{C}$ then the behavior changes. In the first anti-resonance frequency peak during temperature cycle, we also observed PWAS impedance signature attempting to recover and move up in both frequency and amplitude at the room temperatures until the temperature of $200^{\circ} \mathrm{C}$, it no longer recovers after $200{ }^{\circ} \mathrm{C}$ which is close enough to the Curie temperature for PWAS to possess distinct behavior. The degradation of peak shape after $200{ }^{\circ} \mathrm{C}$ is consistent with the change in capacitance behavior. Downward trend in frequency is common among PWAS-EMIS over elevated temperatures even though the shapes of trend are not consistent among samples.

\section{ACKNOWLEDGMENT}

The following financial support agencies are thankfully acknowledged: National Science Foundation Grant \# CMS-0925466; Office of Naval Research \# N00014-11-1-0271, Dr. Ignacio Perez, Technical Representative; Air Force Office of Scientific Research \#FA9550-11-1-0133, Dr. David Stargel, Program Manager; Nuclear Energy University Program Grant \# DENE0000726, Kenny Osborne, Project Manager.

\section{REFERENCES}

1. Lin B, Giurgiutiu V. Modeling of Power and Energy Transduction of Embedded Piezoelectric Wafer Active Sensors for Structural Health Monitoring. 2010;7981:76472P - 76472P - 12. doi:10.1117/12.880120.

2. Giurgiutiu V, Bao J, Zhao W. Active Sensor Wave Propagation Health Monitoring of Beam and Plate Structures. In: Proc of SPIE's 8th International Symposium on Smart Structures and Materials. Newport Beach, CA; 2001.

3. Giurgiutiu V, Zagrai A. Damage Detection in Simulated Aging-Aircraft Panels Using The ElectroMechanical Impedance Technique. In: Adaptive Structures and Material Systems Symposium, ASME Winter Annual Meeting. Orlando, FL; 2000:1-10.

4. Giurgiutiu V, Zagrai a., Jing Bao J. Piezoelectric Wafer Embedded Active Sensors for Aging Aircraft Structural Health Monitoring. Struct Heal Monit. 2002;1(1):41-61. doi:10.1177/147592170200100104.

5. Zagrai AN, Giurgiutiu V. Electro-Mechanical Impedance Method for Damage Identification in Circular Plates. 2001;40. 
6. Liang C, Sun FP, Rogers C a. Coupled Electro-Mechanical Analysis of Adaptive Material Systems -Determination of the Actuator Power Consumption and System Energy Transfer. J Intell Mater Syst Struct. 1994;5(1):12-20. doi:10.1177/1045389X9400500102.

7. Sun FP, Liang C, Rogers CA. Structural modal analysis using collocated piezoelectric actuator/sensors: an electromechanical approach. In: Proc. SPIE 2190, Smart Structures and Materials 1994: Smart Structures and Intelligent Systems, 238. Orlando, FL; 1994.

8. Wolf $\mathrm{R}$ a. Temperature dependence of the piezoelectric response in lead zirconate titanate films. J Appl Phys. 2004;95(3):1397. doi:10.1063/1.1636530.

9. Raghavan a., Cesnik CES. Effects of Elevated Temperature on Guided-wave Structural Health Monitoring. J Intell Mater Syst Struct. 2008;19(12):1383-1398. doi:10.1177/1045389X07086691.

10. Zagrai AN. Piezoelectric Wafer Active Sensor Electro-Mechanical Impedance Structural Health Monitoring. 2002:40-52. http://onlinelibrary.wiley.com/doi/10.1002/cbdv.200490137/abstract. Accessed August 29, 2012. 